\title{
WALKING BIOMECHANICS AND ENERGETICS OF INDIVIDUALS WITH A VISUAL IMPAIRMENT: A PRELIMINARY REPORT
}

original paper

( ) University School of Physical Education in Wroclaw

DOI: https://doi.org/10.5114/hm.2019.85094

\section{HUNTER J. BENNETT ${ }^{1}$, KEVIN A. VALENZUELA ${ }^{2}$, KRISTINA FLEENOR ${ }^{1}$, STEVEN MORRISON ${ }^{3}$, JUSTIN A. HAEGELE ${ }^{1}$}

${ }^{1}$ Department of Human Movement Sciences, Old Dominion University, Norfolk, USA

${ }^{2}$ Department of Kinesiology, California State University Long Beach, Long Beach, CA, USA

${ }^{3}$ School of Physical Therapy and Athletic Training, Old Dominion University, Norfolk, USA

\section{ABSTRACT}

Purpose. Although walking gait in sighted populations is well researched, few studies have investigated persons with visual impairments (VIs). Given the lack of physical activity in people with VIs, it is possible that reduced efficiency in walking could adversely affect activity. The purposes of this preliminary study were to (1) examine the biomechanics and energetics utilized during independent and guided walking in subjects with VIs, and (2) compare gait biomechanics between people with VIs and sighted controls.

Methods. Three-dimensional motion capture and force platforms were used during independent and guided walking at self-selected speeds. Joint angles, moments, external work, and recovery were compared.

Results. The VI group performed independent walking slower and with reduced stride lengths compared with guided walking and sighted controls. Hip range of motion and peak joint moments were reduced during independent walking in the VI group compared with guided walking and controls. Work was greater by $114 \%, 32 \%$, and $16 \%$ in the VI group during independent than during guided walking. Recovery was $11 \%$ greater in guided vs. independent walking.

Conclusions. In the presented preliminary study among 3 persons with congenital VIs, independent walking was a less efficient mode of walking compared with guided walking and that of sighted controls.

Key words: visual impairment, gait, energetics, walking, lower extremity biomechanics

\section{Introduction}

Gait is a common motor task that most individuals perform on a daily basis with relative ease despite challenges such as obstacles to overcome, some other person to avoid, and changes in terrain. Adequate vision is central to optimal gait performance as it allows individuals to recognize these challenges in real time and respond appropriately [1]. For persons with visual impairments (VIs), including those with low vision and complete blindness, performing activities of daily living that involve locomotion can be problematic given the prominent role visual feedback and processing play in many movements. Indeed, adults with VIs tend not to engage in health-enhancing levels of physical activity [2, 3]. Although research has evaluated the physi- cal activity behaviours of individuals with VIs [2], less is known about factors that influence these behaviours. Walking may be particularly relevant to examine, as it is a foundational skill needed for advanced physical activities and has been identified as the most favoured physical activity among this population [4].

Although walking is typically performed with little direct conscious effort, it is considered a multi-joint and multi-system task that requires complex rhythmic coordination of the musculoskeletal system and feedback from the senses. Vision is a critical source of feedback for locomotion, providing information on the surrounding environment and the spatial relationships of body segments [5, 6]. In sighted populations, walking speed is strongly associated with step length, cadence, joint angles, external forces, and muscle activity [7, 8],

Correspondence address: Hunter J. Bennett, Department of Human Movement Sciences, 2016 Student Recreation Center, Old Dominion University, Norfolk, VA 23529, USA, e-mail: hjbennet@odu.edu

Received: November 29, 2018

Accepted for publication: April 10, 2019

Citation: Bennett HJ, Valenzuela KA, Fleenor K, Morrison S, Haegele JA. Walking biomechanics and energetics of individuals with a visual impairment: a preliminary report. Hum Mov. 2019;20(4):8-18; https://doi.org/10.5114/hm.2019.85094. 
which may all be impacted by visual feedback. When visual input is impaired, changes in a person's gait pattern can be seen, including walking at reduced speeds, increasing difficulty navigating obstacles, and increasing metabolic cost $[6,9,10]$.

Except for assessments of speed, little is known about the similarities, or lack thereof, in walking biomechanics (kinematics, kinetics, and energetics) between individuals who are sighted and those with VIs. Within the current literature, only one study performed a biomechanical assessment (joint kinematics and spatiotemporal evaluation) of gait in persons with a range of VIs (including those with low vision and complete blindness) [11]. Although kinematic differences relating to trunk and ankle motion were reported between individuals with VIs and those that were sighted, no assessments were made concerning kinetics or energetics [12]. Joint loads (forces, moments, and powers) provide important information regarding the efforts of individual muscle groups and the body as a whole to perform the given task. Energetics, from a mechanical perspective, can provide insight into the effectiveness of the musculoskeletal system to raise and progress the body during integral tasks such as walking [13]. Additionally, little information is available concerning gait mechanics of the varying modes of walking used by persons with VIs (e.g. with human or animal guides, with long canes, or independent). Persons with low vision may choose similar walking speeds with and without human guides [14]. However, no biomechanical studies have been conducted that would assess walking with and without guides. Therefore, additional research is required to fully describe gait mechanics among individuals with VIs.

For typical walking in sighted populations, 1.11$1.4 \mathrm{~m} / \mathrm{s}$ is the most efficient speed to optimize performance of both the musculoskeletal and cardiovascular systems $[13,14,16]$. Reductions in walking efficiency can be due to impaired muscular efficiency (mechanical work), requiring increases in cardiovascular effort [13], and ultimately could affect motivation for walking for exercise. Recovery, the percent of kinetic and potential energy exchanged during locomotion, is also highest near the most economical walking speed (speed of least work) for sighted individuals [16].

While the mechanics and energetics of walking in sighted populations is well understood, the current knowledgebase of walking in individuals with VIs is limited. Specifically, the relationship between spatiotemporal characteristics, joint biomechanics, and mechanical work among persons with VIs is unknown. Therefore, the purposes of this preliminary study were to (1) examine the kinematics, kinetics, and mechanical work used during 2 common modes of walking (independent and guided) in persons with a VI; and (2) compare gait biomechanics between subjects with VIs and sighted controls.

\section{Material and methods}

\section{Subjects}

Persons with a VI that resulted in complete blindness (i.e., no light perception in either eye) were recruited from the surrounding geographic area to participate in the study. All subjects had congenital VIs. Over an 11-month period, 3 subjects consented to the study: 1 male youth (MY; age: 13 years, mass: $81.46 \mathrm{~kg}$, height: $1.5 \mathrm{~m}$, body mass index [BMI]: $36.2 \mathrm{~kg} / \mathrm{m}^{2}$ ), 1 female young adult (FYA; age: 23 years, mass: $101.16 \mathrm{~kg}$, height: $1.72 \mathrm{~m}, \mathrm{BMI}: 34.2 \mathrm{~kg} / \mathrm{m}^{2}$ ), and $1 \mathrm{fe}-$ male adult (FA; age: 56 years, mass: $83.59 \mathrm{~kg}$, height: $1.65 \mathrm{~m}$, BMI: $30.7 \mathrm{~kg} / \mathrm{m}^{2}$ ). In addition, a control group of 20 sighted subjects (age: $23 \pm 3.8$ years, mass: $73.12 \pm$ $11.8 \mathrm{~kg}$, height: $1.7 \pm 0.1 \mathrm{~m}$, BMI: $25.3 \pm 3.5 \mathrm{~kg} / \mathrm{m}^{2}$, males: 7, females: 13) from a previously published database [17] were included in the study analyses to provide a reference dataset. Exclusion criteria for all study subjects (i.e., those with and without VIs) were the following: (a) any self-reported injury in the last 6 months, (b) prior major joint surgery, (c) any joint replacement, and (d) any joint arthritis.

\section{Experimental protocol}

An 8-camera motion capture system $(200 \mathrm{~Hz}$, Vicon Motion Analysis Inc.) and 3 in-line force platforms (2000 Hz, Bertec FP-4060) were used to collect 3-dimensional kinematic and ground reaction force (GRF) data during level walking. The subjects wore spandex shorts and standard laboratory tennis shoes. Reflective motion capture markers were placed bilaterally on the acromion processes, anterior and posterior superior iliac spines, greater trochanters, medial and lateral epicondyles, medial and lateral malleoli, and metatarsal heads. Clusters of 4 markers were attached to the trunk, posterior pelvis, thighs, shanks, and shoe heels to track 8 segments.

Both VI and control groups performed a 5-minute warm-up on a treadmill walking at a self-selected speed, followed by several level walking warm-up trials until the subject was familiarized with the 18-meter walkway (Figure 1). The walkway was constructed to allow for collecting GRFs during 3 continuous steps, 
H. Bennett et al., Gait biomechanics in visual impairment

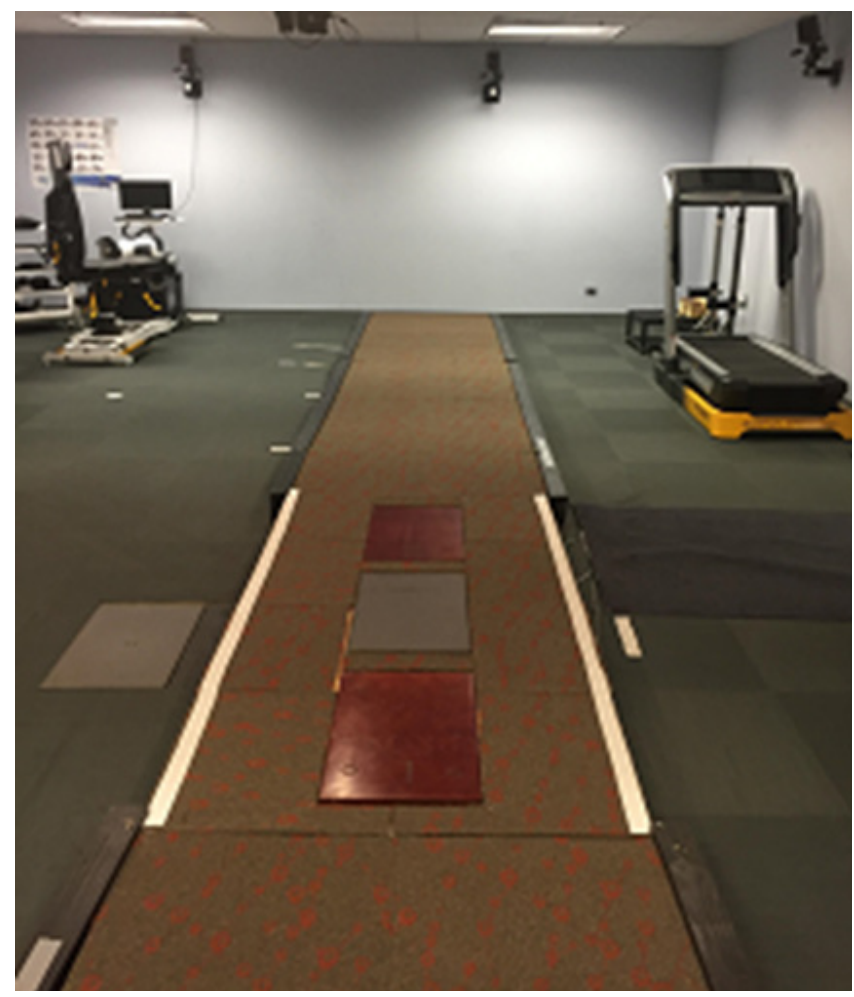

Figure 1. Walkway used for 3-dimensional motion capture. The participant walked independently and with a human guide on the raised platform. Researchers were present alongside the platform during walking for participant safety. Three in-line force platforms captured ground reaction forces

a requirement for determining bilateral limb work. During warm-up trials, the subjects' self-selected speeds were recorded with electronic timing gates and used to determine average self-selected speeds. The VI group performed 5 successful trials in each of the 2 walking conditions: independently using their own walking cane and with a human guide. The VI group was instructed to walk at their own self-selected speeds for both walking conditions. For guided walking, an experienced sighted guide walked on the preferred side of the subject with the subject's hand placed on the guide's elbow and a half step behind the guide. The guide reminded the subject during each trial that the subject was to set the walking speed [14]. In all walking conditions, researchers were present with the VI subjects to ensure safety on the walkway. In the VI group, walking speeds were dependent on the comfort level of the subjects, and thus were not standardized between walking conditions. In both VI and control groups, the subjects performed 5 successful trials of level walking at $\pm 5 \%$ of their average self-selected speeds. A successful trial included full foot contact on the force plates.
Data analyses

Three-dimensional kinematics and GRFs were imported into the Visual3D software (version 5, C-Motion Inc.) and filtered at $8 \mathrm{~Hz}$ [18]. An 8-segment model [17] was constructed from marker positions. An X-Y-Z (extension-adduction-rotation) Cardan rotation sequence and the right hand rule were used for hip [19, 20], knee, and ankle kinematics and kinetics computations. Internal joint moments were normalized to body mass $(\mathrm{Nm} / \mathrm{kg})$. Speed, anterior centre of mass displacement (stride length), vertical centre of mass displacement, and sagittal plane ankle, knee, and hip range of motion (ROM) (min to max) and peak moments were chosen as variables of interest.

External mechanical work was calculated as the sum of the positive increments of external energy during a full stride with the use of previously determined methods [13, 16, 21], which are briefly described here. External energy is the sum of the potential and kinetic energies used to raise and translate the model's centre of mass. Three-dimensional centre of mass accelerations were obtained from the raw GRFs and the subjects' mass [13, 16, 21]. From the centre of mass accelerations, instantaneous kinetic and potential energies were computed and then summed to derive external energy. Positive increments of external energies for each stride were summed to obtain total external mechanical work. External mechanical work was expressed as: $\mathrm{J} / \mathrm{mass}, \mathrm{J} / \mathrm{mass}^{*}$ stride length ( $\left.\mathrm{J} / \mathrm{kgm}\right)$, and $\mathrm{J} / \mathrm{mass}^{*}$ speed $\left(\mathrm{J} / \mathrm{kgms}^{-1}\right)$. Recovery, the percent of mechanical energy exchange between kinetic and potential energies during locomotion, was also calculated [15, 16]:

$$
\text { Recovery }(\%)=100 \cdot \frac{\left|\mathrm{W}_{\mathrm{k}}\right|+\left|\mathrm{W}_{\mathrm{p}}\right|-\left|\mathrm{W}_{\text {Ext }}\right|}{\left|\mathrm{W}_{\mathrm{k}}\right|+\left|\mathrm{W}_{\mathrm{p}}\right|}
$$

Recovery compares the maximum possible work without exchange of positive kinetic $\left(\mathrm{W}_{\mathrm{k}}\right)$ and potential $\left(W_{p}\right)$ energy and the work actually done $\left(W_{\text {Ext }}\right)$. Maximum values for recovery in sighted persons are ca. $65 \%$ between 1.1 and $1.4 \mathrm{~m} / \mathrm{s}[15,16]$.

Statistical comparisons of between-group differences (VI and control) were performed with nonparametric Mann-Whitney tests, with the significance level set at 0.05. Comparisons of independent and guided walking conditions within the VI group could not be performed owing to the small sample size. Given the preliminary study sample size $(n=3)$, the smallest achievable significance level between 2 related groups is 0.100 . Absolute (e.g., degrees and $\mathrm{Nm}$ ) and relative 
(percent differences) differences were reported for independent and guided walking conditions. Coefficients of determination (standard deviation/mean*100) for joint moments are also presented for both groups and conditions. In consideration of walking speed differences between groups, we also normalized stride length, work (J/kgm), and recovery to existing datasets with sighted individuals walking at similar speeds $[16,22]$.

\section{Ethical approval}

The research related to human use has complied with all the relevant national regulations and institutional policies, has followed the tenets of the Declaration of Helsinki, and has been approved by the Old Dominion University review board.

\section{Informed consent}

Informed consent has been obtained from all individuals included in this study.

\section{Results}

Persons with VIs walked significantly slower and had significantly reduced stride lengths during both independent and guided walking compared with the control group (both $p=0.006$; Table 1 ). No differences were found in vertical displacement between groups $(p>0.05)$. For all subjects with VIs, the preferred walking speed was reduced during independent when compared with guided walking (Table 1).

Knee biomechanics were not different between the control group and either walking condition for the VI group (all $p>0.05$; Table 2). Ankle and hip ROMs were significantly reduced in independent and guided walking compared with controls (all $p<0.05$; Table 2). Overall, ROMs tended to differ by $<2^{\circ}$ between independent and guided conditions (Table 2), except for hip ROM for the young male (independent reduced by $11^{\circ}$ ). Peak ankle and hip moments were significantly reduced during independent and guided walking com-

Table 1. Speed, vertical displacement, and stride length comparisons: mean $\pm S D$

\begin{tabular}{lccccccccc}
\hline \multirow{2}{*}{ Variable } & \multicolumn{2}{c}{ MY } & \multicolumn{2}{c}{ FYA } & \multicolumn{2}{c}{ FA } & \multicolumn{2}{c}{ Control } & \multicolumn{2}{c}{$p$ value } \\
\cline { 2 - 9 } & Independent & Guided & Independent & Guided & Independent & Guided & Normal & I-C & G-C \\
\hline Speed (m/s) & $0.72 \pm 0.11$ & $1.23 \pm 0.01$ & $0.66 \pm 0.04$ & $0.77 \pm 0.04$ & $0.85 \pm 0.02$ & $1.22 \pm 0.02$ & $1.60 \pm 0.14$ & 0.006 & 0.006 \\
COM vertical & $0.03 \pm 0.00$ & $0.03 \pm 0.01$ & $0.04 \pm 0.01$ & $0.04 \pm 0.00$ & $0.04 \pm 0.00$ & $0.04 \pm 0.00$ & $0.01 \pm 0.08$ & 0.492 & 0.501 \\
$\begin{array}{l}\text { displacement (m) } \\
\text { Stride length (m) }\end{array}$ & $1.04 \pm 0.04$ & $1.27 \pm 0.04$ & $0.89 \pm 0.08$ & $0.92 \pm 0.01$ & $0.86 \pm 0.02$ & $1.06 \pm 0.02$ & $1.60 \pm 0.09$ & 0.006 & 0.006 \\
\hline
\end{tabular}

SD - standard deviation, MY - male youth, FYA - female young adult, FA - female adult, I-C - nonparametric test significance level between independent walking and control group, G-C - nonparametric test significance level between guided walking and control group, COM - centre of mass

Table 2. Sagittal plane ankle, knee, and hip joint ROMs and moments: mean $\pm S D$

\begin{tabular}{|c|c|c|c|c|c|c|c|c|c|}
\hline \multirow{2}{*}{ Variable } & \multicolumn{2}{|c|}{ MY } & \multicolumn{2}{|c|}{ FYA } & \multicolumn{2}{|c|}{ FA } & \multirow{2}{*}{$\begin{array}{l}\text { Control } \\
\text { Normal }\end{array}$} & \multicolumn{2}{|c|}{$p$ value } \\
\hline & Independent & Guided & Independent & Guided & Independent & Gui & & $\mathrm{I}-\mathrm{C}$ & G-C \\
\hline o) & $.41 \pm 6.2$ & 21.86 & $26.0 \pm 2.3$ & $24.7 \pm 2.4$ & $24.1 \pm 0.5$ & $24.1 \pm 0.6$ & $28.1 \pm 3.2$ & 0.045 & 0.022 \\
\hline $\begin{array}{l}\text { Pltflx moment } \\
(\mathrm{Nm} / \mathrm{kg})\end{array}$ & $-0.95 \pm 0.11$ & $-1.31 \pm 0.04$ & $-0.44 \pm 0.08$ & $-0.64 \pm 0.04$ & $-0.68 \pm 0.01$ & $-1.10 \pm 0.00$ & $-1.58 \pm 0.18$ & 0.006 & 0.006 \\
\hline Knee Flx ROM $\left(^{\circ}\right)$ & $46.2 \pm 1.2$ & $46.7 \pm 4.3$ & $49.9 \pm 1.8$ & $48.9 \pm 2.3$ & $53.1 \pm 3.5$ & $54.8 \pm 1.7$ & $47.0 \pm 3.3$ & 0.411 & 0.411 \\
\hline $\begin{array}{l}\text { Knee Ext moment } \\
(\mathrm{Nm} / \mathrm{kg})\end{array}$ & $0.44 \pm 0.09$ & $0.81 \pm 0.06$ & $0.65 \pm 0.12$ & $0.87 \pm 0.13$ & $0.77 \pm 0.07$ & $0.60 \pm$ & $0.71 \pm 0.24$ & 0.465 & 0.715 \\
\hline Hip Ext ROM $\left(^{\circ}\right)$ & $24.2 \pm 6.8$ & $00.1 \pm 2.4$ & $29.8 \pm 3.7$ & $34.5 \pm$ & $32.1 \pm 5.8$ & $33.8 \pm$ & $47.6 \pm 7.0$ & 0.006 & 0.006 \\
\hline $\begin{array}{l}\text { Hip Ext moment } \\
(\mathrm{Nm} / \mathrm{kg})\end{array}$ & $-0.45 \pm 0.13$ & $-0.53 \pm 0.04$ & $-0.51 \pm 0.08$ & $-0.52 \pm 0.09$ & $-0.45 \pm 0.08$ & $-0.64 \pm 0.05$ & $-0.85 \pm 0.16$ & 0.006 & 0.018 \\
\hline
\end{tabular}

ROM - range of motion ( $\max$ - min), SD - standard deviation, MY - male youth, FYA - female young adult, FA - female adult, I-C - nonparametric test significance level between independent walking and control group, G-C - nonparametric test significance level between guided walking and control group, Drflx - dorsiflexion, Pltflx - plantarflexion, Flx - flexion, Ext - extension; moments are internal moments 
H. Bennett et al., Gait biomechanics in visual impairment

Table 3. Mechanical work comparisons between walking conditions and groups: mean $\pm S D$

\begin{tabular}{|c|c|c|c|c|c|c|c|c|c|}
\hline \multirow{2}{*}{ Variable } & \multicolumn{2}{|c|}{ MY } & \multicolumn{2}{|c|}{ FYA } & \multicolumn{2}{|c|}{ FA } & \multirow{2}{*}{$\begin{array}{c}\text { Control } \\
\text { Normal }\end{array}$} & \multicolumn{2}{|c|}{$p$ value } \\
\hline & Independent & Guided & Independent & Guided & Independent & Guided & & $\mathrm{I}-\mathrm{C}$ & G-C \\
\hline Work (J/kg) & $0.55 \pm 0.11$ & $0.43 \pm 0.01$ & $0.43 \pm 0.02$ & $0.40 \pm 0.03$ & $0.40 \pm 0.06$ & $0.48 \pm 0.05$ & $0.53 \pm 0.14$ & 0.273 & 0.201 \\
\hline Work (J/kgm) & $0.52 \pm 0.09$ & $0.34 \pm 0.02$ & $0.61 \pm 0.03$ & $0.54 \pm 0.04$ & $0.49 \pm 0.07$ & $0.47 \pm 0.04$ & $0.33 \pm 0.08$ & 0.009 & 0.077 \\
\hline Work $\left(\mathrm{J} / \mathrm{kgms}^{-1}\right)$ & $0.77 \pm 0.08$ & $0.36 \pm 0.02$ & $0.83 \pm 0.01$ & $0.63 \pm 0.09$ & $0.57 \pm 0.08$ & $0.49 \pm 0.05$ & $0.33 \pm 0.09$ & 0.013 & 0.127 \\
\hline Recovery (\%) & $43.4 \pm 10.4$ & $48.0 \pm 5.7$ & $47.9 \pm 3.0$ & $59.8 \pm 7.1$ & $50.1 \pm 3.8$ & $58.9 \pm 6.1$ & $53.5 \pm 8.5$ & 0.022 & 0.008 \\
\hline
\end{tabular}

SD - standard deviation, MY - male youth, FYA - female young adult, FA - female adult, I-C - nonparametric test significance level between independent walking and control group, G-C - nonparametric test significance level between guided walking and control group

Table 4. Sagittal plane ankle, knee, and hip joint coefficients of determination (\%)

\begin{tabular}{|c|c|c|c|c|c|c|c|}
\hline \multirow{2}{*}{ Variable } & \multicolumn{2}{|c|}{ MY } & \multicolumn{2}{|c|}{ FYA } & \multicolumn{2}{|c|}{ FA } & \multirow{2}{*}{$\frac{\text { Control }}{\text { Norma }}$} \\
\hline & Independent & Guided & Independent & Guided & Independent & Guided & \\
\hline Drflx ROM & 31.9 & 5.9 & 8.8 & 9.7 & 2.1 & 2.5 & 11.4 \\
\hline Pltflx moment & 11.6 & 3.1 & 18.2 & 6.3 & 1.5 & 0.0 & 11.4 \\
\hline Knee Flx ROM & 2.6 & 9.2 & 3.6 & 4.7 & 6.6 & 3.1 & 7.0 \\
\hline Knee Ext moment & 20.5 & 7.4 & 18.5 & 14.9 & 9.1 & 15.0 & 33.8 \\
\hline Hip Flx ROM & 28.1 & 6.8 & 12.4 & 4.6 & 18.1 & 2.1 & 14.7 \\
\hline Hip Ext moment & 28.9 & 7.5 & 15.7 & 17.3 & 17.8 & 7.8 & 18.8 \\
\hline
\end{tabular}

MY - male youth, FYA - female young adult, FA - female adult, Drflx - dorsiflexion, ROM - range of motion,

Pltflx - plantarflexion, Flx - flexion, Ext - extension; moments are internal moments

Table 5. Stride length, work, and recovery normalized (z-scores) with speed-matched data from the literature

\begin{tabular}{lcccccc}
\hline \multirow{2}{*}{ Variable } & \multicolumn{3}{c}{ MY } & \multicolumn{2}{c}{ FYA } & FA \\
\cline { 2 - 7 } & Independent & Guided & Independent & Guided & Independent & Guided \\
\hline Stride length & 0.2 & -0.2 & -1.0 & -1.2 & -2.1 & -1.9 \\
Work (J/kgm) & 7.1 & 1.1 & 11.6 & 8.1 & 5.6 & 4.4 \\
Recovery & -0.6 & -1.9 & -0.1 & 1.3 & 0.2 & 0.2 \\
\hline
\end{tabular}

MY - male youth, FYA - female young adult, FA - female adult; stride length compared with [22], work and recovery compared with [16]

pared with controls (all $p<0.05$; Table 2). Ankle moments tended to increase from independent to guided walking for all subjects with VIs. Knee moments generally increased for MY and FYA (by an average of $0.29 \mathrm{Nm} / \mathrm{kg}$ ), but decreased for FA (by $0.17 \mathrm{Nm} / \mathrm{kg}$ ). Hip moments only increased for MY and FA in guided compared with independent walking (average: 0.14 $\mathrm{Nm} / \mathrm{kg}$ ).

Mechanical work per mass $(\mathrm{J} / \mathrm{kg})$ was not significantly different between independent or guided conditions and the control group ( $p>0.05$; Table 3). Work normalized to mass* stride length $(\mathrm{J} / \mathrm{kgm})$ and to mass*velocity $\left(\mathrm{J} / \mathrm{kgms}^{-1}\right)$ was significantly increased during independent walking compared with controls ( $p=0.009$ and $p=0.013$, respectively; Table 3), but not during guided walking compared with controls
( $p=0.077$ and $p=0.127$, respectively). Recovery (\%) was significantly decreased for both independent and guided walking compared with controls $(p=0.022$ and $p=0.008$, respectively; Table 3). Markedly less work per mass and velocity was performed during guided compared with independent walking for all subjects with VIs (range: 16-113\%). Recovery increased by $>10 \%$ in all subjects with VIs during guided compared with independent walking (Table 3). Coefficients of determination and are presented in Table 4.

Normalized stride length, work (J/kgm), and recovery are presented in Table 5. When normalized, stride lengths do not appear different between groups (all average $\mathrm{z}$-scores below \pm 1.96 ) or between conditions for the VI group (z-scores differ by < 0.3). Similarly, normalized recovery does not appear different 
between conditions for the VI group (all below \pm 1.96 ). However, normalized work (J/kgm) was generally much lower in guided compared with independent walking (by 1.2 to 6 units) and for the control group compared with the VI group.

\section{Discussion}

The purposes of this preliminary study were to (1) examine the kinematics, kinetics, and mechanical work used during independent and guided walking in persons with a congenital VI; and (2) compare gait biomechanics between people with VI and a cohort of sighted controls. This is a first effort in describing the gait mechanics of persons with a VI in 2 of the most used forms of locomotion. As such, this study is limited in that the subject pool for the VI group was small (3 subjects). Therefore, future research is certainly required to obtain larger subject pools to make inferences about persons with VIs. However, some important trends between groups and locomotion tasks can be found in this preliminary report.

As expected, spatiotemporal and joint level biomechanics significantly differed between the VI and control groups, as well as within the VI group during independent and guided walking. Most spatiotemporal and joint biomechanics differences are likely linked with the markedly reduced self-selected speeds during independent walking. However, the VI group independent walking was a much less efficient mode of locomotion compared with VI group guided walking and control group walking. Interestingly, guided walking work per mass*stride length and recovery approached those of the sighted control group.

\section{Spatial and temporal characteristics}

Speed and stride lengths were significantly reduced in both independent and guided walking conditions in the VI group compared with the controls. Average gait speeds were by $70 \%$ (MY), $17 \%$ (FYA), and 44\% (FA) larger during guided compared with independent walking (Table 1) among VI subjects. Stride lengths during guided walking were also larger than during independent walking: by 22\% (MY), 3\% (FYA), and $23 \%$ (FA). As walking speeds were self-selected on the basis of subjects' safety and comfort level, differences in this parameter could be expected. Additionally, walking speeds and stride lengths were reduced in the VI group compared with sighted controls within this study. A previous comparison of sighted guide and independent walking in persons with low vision (generally older adults with age- or trauma-induced impairments) found no differences in the preferred walking speed [14]. Differences between our findings and the previous study may stem from the length in time of vision loss, as those with congenital VIs tend to walk more slowly than those who are late-blind [10].

\section{Joint ROM and peak joint moments}

Interestingly, waveform patterns of joint rotations and moments (but not peak values) appear to be quite similar between the conditions (FYA lower extremity dynamics presented in Figure 2a, b) and are visually similar to those reported in sighted adults of similar age $[23,24]$. The similarities in movement and joint loading patterns between the conditions suggest that guidance did not greatly impact on the fluidity of lower extremity gait mechanics. Additionally, the similarity in movement patterns between our subjects with VIs and sighted young adults [23, 24] suggests that normal gait mechanics is achieved without governance of visual input. Differences in joint ROM between the conditions were quite small $\left(<5^{\circ}\right)$; however, up to ca. $10^{\circ}$ differences were found in hip ROM for one subject (MY). General increases in joint moments during guided compared with independent walking and between VI and control groups were expected, given the increased gait speeds during guided walking and in the control group. Interestingly, variations in hip, knee, and ankle peak joint moments and ankle and hip ROM were larger during independent compared with guided walking (Table 4). In addition, variations in most joint biomechanics decreased in the guided condition to or below the control group levels. This increase in the variability of lower extremity joint loads during independent walking suggests that independent walking may not be as efficient as guided walking in persons with VIs.

\section{Mechanical work and recovery}

Mechanical work provides an assessment of the energy required to perform the given task, with larger positive mechanical work indicating more muscle work required to move [15, 21]. Work per mass was increased during guided compared with independent walking and in the control compared with VI group (Table 3), which should be expected owing to increased displacement and walking speeds during guided walking and in controls compared with subjects with VIs (Table 1). As no previous study has assessed work or gait biomechanics in VI subjects or between their 


\section{HUMAN MOVEMENT}

H. Bennett et al., Gait biomechanics in visual impairment

$2 a$
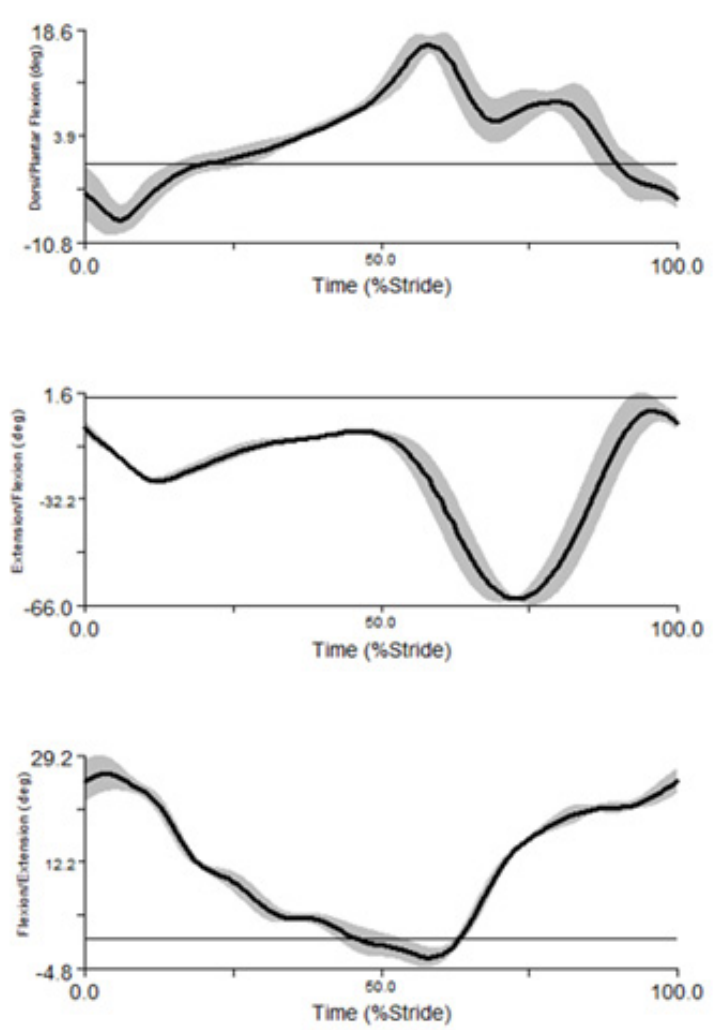

$2 b$
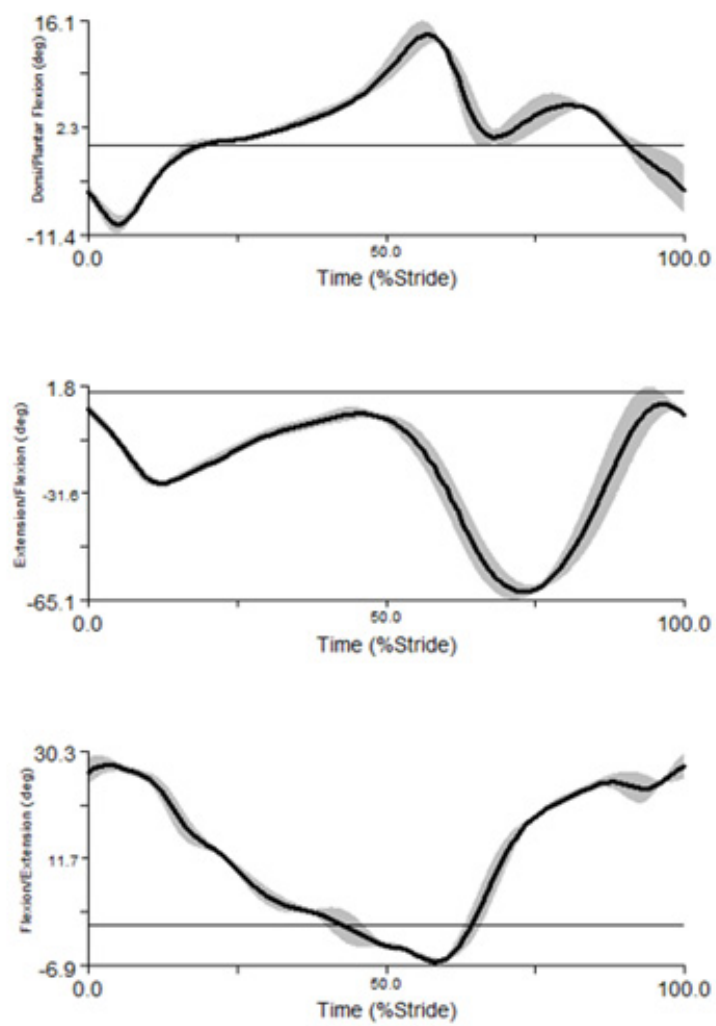
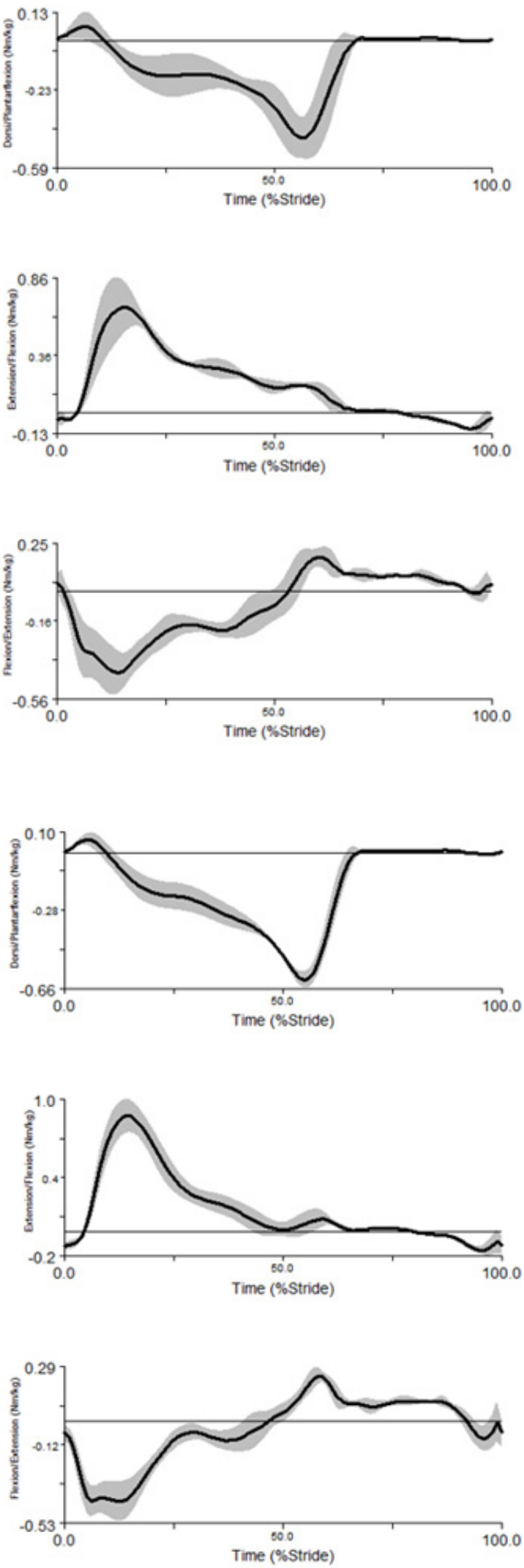

Figure 2. Joint kinematics and kinetics of the female young adult during (a) independent walking and (b) guided walking. Top row: sagittal plane ankle angles and moments; middle row: sagittal plane knee angles and moments; bottom row: sagittal plane hip angles and moments. Kinematics and kinetics are represented as mean (black line) and one standard deviation (shaded region) from heel contact to subsequent heel contact (\% stride) 

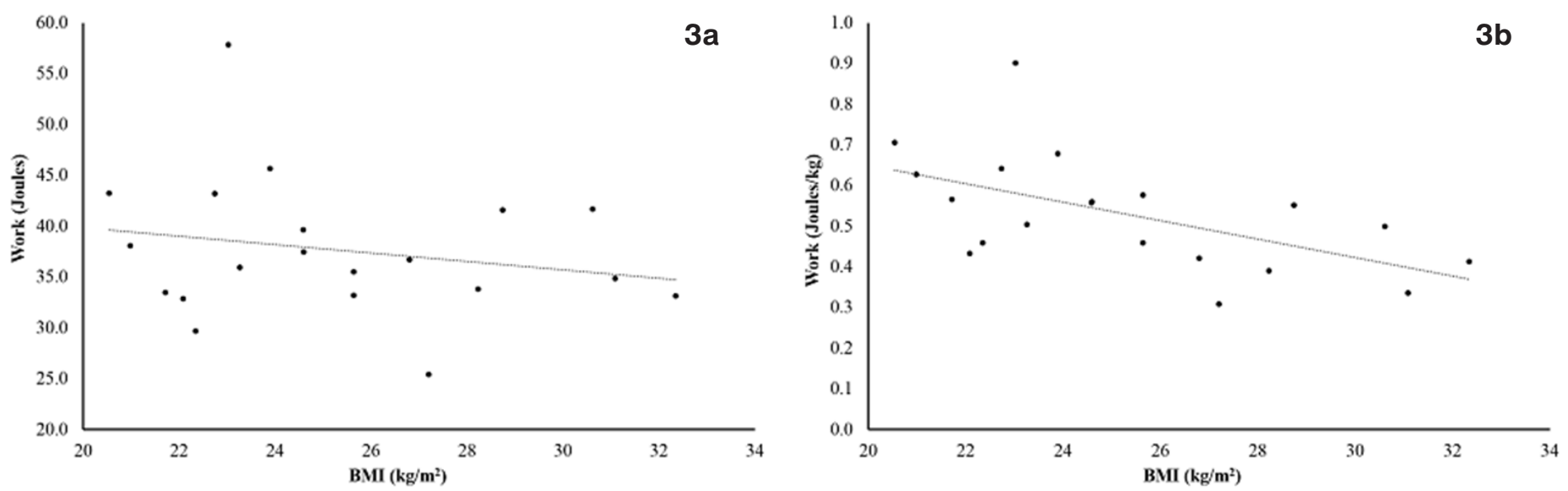

Figure 3. Relationship between BMI and external work in sighted controls. (a) BMI has no relationship (r2 = 0.04) with non-normalized external work. (b) BMI presents a moderate inverse relationship with external work normalized to body mass $(\mathrm{r} 2=0.32)$

common modes of locomotion, relations/comparisons can only be made with those reported from clinical and healthy populations $[13,25,26]$. Similar to these previous assessments, gait speed, stride length, and vertical displacements play a significant role during walking. Therefore, mechanical work must be examined not only normalized to mass, but also to the differences in spatiotemporal variables between walking conditions and groups.

Mechanical work per mass*stride length found in our sighted subjects are similar to previous reports in healthy [16] and clinical [13, 25, 26] populations. However, work per mass* ${ }^{*}$ stride length was increased among our subjects with VIs during independent walking compared with our sighted health controls and previous studies [13, 25, 26]. The differences in effective muscle work per stride (external mechanical work) observed here illustrate the reduced efficiency of movement in persons with VIs. Previous studies have also demonstrated that mechanical work per distance increases when walking slower or faster than the most efficient speeds of 1.1-1.4 m/s [16, 21]. As such, sighted individuals in these previous assessments typically chose self-selected speeds that were within the mechanical efficiency range. For our subjects with VIs, work per mass*speed was much larger during their slower paced independent compared with their faster paced guided walking. The increased external work required to raise and accelerate the centre of mass during slower paced independent walking suggests that the self-selected independent walking speeds may not be the most efficient for conservation of energy, but could be chosen for caution and stability. Although many factors play a role in physical activity, the greater mechanical cost in independent walking may contribute to the decreased independent physical activity levels in this population.

In addition to walking speed, excessive mass or larger BMI might influence walking efficiency. Our subjects with VIs had higher BMIs (range: $31-36 \mathrm{~kg} / \mathrm{m}^{2}$ ) than the sighted control group $\left(20-32 \mathrm{~kg} / \mathrm{m}^{2}\right)$, which could explain some of the differences between the groups. However, the 20 sighted subjects' data pointed at no relationship between BMI and external work $\left(\mathrm{r}^{2}=0.04\right.$; Figure 3a) and a moderate inverse relationship with external work normalized to mass $\left(r^{2}=0.32\right.$; Figure $3 \mathrm{~b})$. Therefore, it appears that a greater mass does not necessarily result in greater external work in sighted persons. As we were not able to collect a range of individuals with VIs, future research should consider the effects of age and other anthropometric factors that may affect external work in populations with and without VIs.

Comparisons of recovery showed increased conservation of energy in guided compared with independent walking (Table 3). Because recovery/transfer between potential and kinetic energy increases from slow walking up to the most efficient speed of ca. 1.1-1.4 m/s for sighted individuals $[15,16]$, it is possible that recovery increased in our 3 subjects during guided compared with independent walking owing to an increase in gait speed. However, regardless of the influence of gait speed, the increased recovery in guided walking indicates an improved exchange of kinetic and potential energies, following a more efficient 'inverted pendulum' movement. Therefore, it appears that independent walking was indeed a more demanding and less efficient task than guided walking in our subjects with VIs.

Given the differences in walking speeds between the conditions and groups, it is important to consider 
normalizing our data prior to concluding that effects are condition- and not spatiotemporally-based. Table 5 contains stride length, work $(\mathrm{J} / \mathrm{kgm})$, and recovery variables normalized to 2 published studies with speedmatched walking data [16, 22]. Analyses of the raw data indicate that those with VIs chose to walk faster (still slower than controls), with longer strides, reduced work, and improved recovery with a human guide than during independent walking. The speed-normalized results imply that walking with a human guide reduces mechanical work compared with independent walking, without requiring dramatic changes to stride length. Similarities in normalized recovery suggest that improving the magnitude of work, and not the exchange of energy, is the major advantage of walking with a human guide. Future research with larger datasets should consider including speed-matched groups and conditions.

It is known that individuals with VIs tend not to engage in sufficient levels of physical activity to garner health-related benefits [2,3]. While some research has identified environmental barriers [4] or psychology factors $[27,28]$ that may impede physical activity behaviour, little is known about what factors influence physical activity from a biomechanical perspective. When viewed in its entirety, this study indicates that persons with VIs have less efficient gaits than sighted individuals when walking without a sighted guide. Since walking is largely considered the most favoured physical activity among people with VIs [4], it is logical to suggest that inefficiency in that behaviour may influence physical activity. Thus, future intervention research should seek to implement programs to enhance movement efficiency among individuals with VIs, as well as examine the influence that improved movement efficiency can have on physical activity engagement.

\section{Limitations}

There are several limitations to acknowledge with this work. First, the study has a small VI sample size, so caution is required in generalizing the results. Future studies involving the VI population should certainly include larger subject pools. Second, only sagittal plane biomechanics and energetics (the primary proponents of locomotion) were considered. Further work should examine the frontal and transverse planes. Third, this study did not include BMI matches between groups. The current literature indicates no significant sagittal plane kinematic or kinetic (when normalized to body mass, as in the current study) differences between obese and healthy weight individuals [29-32].
However, additional insights may be obtained by comparing BMI groups within and between populations with and without VIs. Lastly, age is an important consideration when examining gait mechanics. The current study subject pool included an adolescent group (10-19 years) and an adult group (20-59 years) (World Health Organization), which could present age-related issues when comparing between/within groups. However, multiple gait studies indicate that normative mechanics is reached by the age of 7-8 years [33-38]. Thus, the major findings (work and joint biomechanics) from this study are likely not confounded by age discrepancies. The literature is not as clear on spatiotemporal variables and gait maturity, as one large study indicates no differences in adolescents and adults [39], while another suggests that 13-year-olds do not exhibit the spatiotemporal characteristics of 19-year-olds [40]. Given the very small subject pool in this study, future research incorporating age-matched methodology is certainly warranted.

\section{Conclusions}

From this preliminary work, it appears that persons with a congenital VI walk more slowly. In general, only spatiotemporal parameters and peak joint moments were markedly different between conditions, which are linked with walking speed. However, independent walking required greater mechanical work per walking speed, suggesting this mode of walking required additional muscular effort and may not be as efficient as walking with a guide. Additionally, compared with sighted controls, the VI groups' gait speeds, ROMs, joint moments, and recovery during independent and guided walking were reduced.

\section{Disclosure statement}

No author has any financial interest or received any financial benefit from this research.

\section{Conflict of interest}

The authors state no conflict of interest.

\section{References}

1. Cromwell RL, Newton RA, Forrest G. Influence of vision on head stabilization strategies in older adults during walking. J Gerontol A Biol Sci Med Sci. 2002; 57(7):M442-M448; doi: 10.1093/gerona/57.7.m442.

2. Marmeleira J, Laranjo L, Marques O, Pereira C. Physical activity patterns in adults who are blind as assed by accelerometry. Adapt Phys Activ Q. 2014;31(3):283296; doi: 10.1123/apaq.2013-0039. 
3. Sadowska D, Krzepota J. Assessment of physical activity of people with visual impairments and individuals who are sighted using the International Physical Activity Questionnaire. J Vis Impair Blind. 2015;109(2): 119-129; doi: 10.1177/0145482X1510900207.

4. Jaarsma EA, Dekker R, Koopsman SA, Dijkstra PU, Geertzen JHB. Barriers to and facilitators of sports participation in people with visual impairments. Adapt Phys Activ Q. 2014;31(3):240-264; doi: 10.1123/20130119.

5. Faquin BS, Candido CRC, Mochizuki L, Okazaki VHA. Effect of visual and vestibular information on spatial perception on gait. Hum Mov. 2018;19(2):39-45; doi: 10.5114/hm.2018.74058.

6. Patla AE. How is human gait controlled by vision. Ecol Psychol. 1998;10(3-4):287-302; doi: 10.1080/10407413. 1998.9652686.

7. Chiu MC, Wang MJ. The effect of gait speed and gender on perceived exertion, muscle activity, joint motion of lower extremity, ground reaction force and heart rate during normal walking. Gait Posture. 2007;25(3):385392; doi: 10.1016/j.gaitpost.2006.05.008.

8. Chung MJ, Wang MJ. The change of gait parameters during walking at different percentage of preferred walking speed for healthy adults aged $20-60$ years. Gait Posture. 2010;31(1):131-135; doi: 10.1016/j.gaitpost. 2009.09.013.

9. Kobberling G, Jankowski LW, Leger L. Energy cost of locomotion in blind adolescents. Adapt Phys Activ Q. 1989;6(1):58-67; doi: 10.1123/apaq.6.1.58.

10. Nakamura T. Quantitative analysis of gait in the visually impaired. Disabil Rehabil. 1997;19(5):194-197; doi: 10.3109/09638289709166526.

11. Hallemans A, Ortibus E, Meire F, Aerts P. Low vision affects dynamic stability of gait. Gait Posture. 2010; 32(4):547-551; doi: 10.1016/j.gaitpost.2010.07.018.

12. Hallemans A, Beccu S, Van Loock K, Ortibus E, Truijen S, Aerts P. Visual deprivation leads to gait adaptations that are age- and context-specific: II. Kinematic parameters. Gait Posture. 2009;30(3):307-311; doi: 10.1016/j.gaitpost.2009.05.017.

13. Mahaudens P, Detrembleur C, Mousny M, Banse X. Gait in adolescent idiopathic scoliosis: energy cost analysis. Eur Spine J. 2009;18(8):1160-1168; doi: 10.1007/ s00586-009-1002-0.

14. Soong GP, Lovie-Kitchin JE, Brown B. Preferred walking speed for assessment of mobility performance: sighted guide versus non-sighted guide techniques. Clin Exp Optom.2000;83(5):279-282; doi:10.1111/j.1444-0938. 2000.tb05017.x.

15. Cavagna GA, Thys H, Zamboni A. The sources of external work in level walking and running. J Physiol. 1976; 262(3):639-657; doi: 10.1113/jphysiol.1976.sp011613.

16. Willems PA, Cavagna GA, Heglund NC. External, internal and total work in human locomotion. J Exp Biol. 1995;198(Pt 2):379-393.
17. Bennett HJ, Fleenor K, Weinhandl JT. A normative database of hip and knee joint biomechanics during dynamic tasks using anatomical regression prediction methods. J Biomech. 2018;81:122-131; doi: 10.1016/j. jbiomech.2018.10.003.

18. Bennett HJ, Shen G, Cates HE, Zhang S. Effects of toe-in and toe-in with wider step width on level walking knee biomechanics in varus, valgus, and neutral knee alignments. Knee. 2017;24(6):1326-1334; doi: 10.1016/ j.knee.2017.08.058.

19. Bell AL, Brand RA, Pedersen DR. Prediction of hip joint centre location from external landmarks. Hum Mov Sci. 1989;8(1):3-16; doi: 10.1016/0167-9457(89)90020-1.

20. Bell AL, Pedersen DR, Brand RA. A comparison of the accuracy of several hip center location prediction methods. JBiomech. 1990;23(6):617-621; doi: 10.1016/00219290(90)90054-7.

21. Cavagna GA, Kaneko M. Mechanical work and efficiency in level walking and running. J Physiol. 1977; 268(2):467-481; doi: 10.1113/jphysiol.1977.sp011866.

22. Holden JP, Chou G, Stanhope SJ. Changes in knee joint function over a wide range of walking speeds. Clin Biomech. 1997;12(6):375-382; doi: 10.1016/S02680033(97)00020-X.

23. Kerrigan DC, Todd MK, Della Croce U. Gender differences in joint biomechanics during walking: normative study in young adults. Am J Phys Med Rehabil. 1998;77(1):2-7; doi: 10.1097/00002060-19980100000002.

24. Riley PO, Paolini G, Della Croce U, Paylo KW, Kerrigan DC. A kinematic and kinetic comparison of overground and treadmill walking in healthy subjects. Gait Posture. 2007;26(1):17-24; doi: 10.1016/j.gaitpost.2006. 07.003.

25. Detrembleur C, Dierick F, Stoquart G, Chantraine F, Lejeune T. Energy cost, mechanical work, and efficiency of hemiparetic walking. Gait Posture. 2003;18(2): 47-55; doi: 10.1016/S0966-6362(02)00193-5.

26. Detrembleur C, Vanmarsenille JM, De Cuyper F, Dierick F. Relationship between energy cost, gait speed, vertical displacement of centre of body mass and efficiency of pendulum-like mechanism in unilateral amputee gait. Gait Posture. 2005;21(3):333-340; doi: 10.1016/j.gaitpost.2004.04.005.

27. Haegele JA, Hodge SR, Kozub FM. Beliefs about physical activity and sedentary behaviors of adults with visual impairments. Disabil Health J. 2017;10(4):571579; doi: 10.1016/j.dhjo.2017.03.008.

28. Haegele JA, Kirk TN, Zhu X. Self-efficacy and physical activity among adults with visual impairments. Disabil Health J. 2018;11(2):324-329; doi: 10.1016/j. dhjo.2017.10.012.

29. Browning RC, Kram R. Effects of obesity on the biomechanics of walking at different speeds. Med Sci Sports Exerc. 2007;39(9):1632-1641; doi: 10.1249/mss.0b013 e318076b54b. 


\section{HUMAN MOVEMENT}

H. Bennett et al., Gait biomechanics in visual impairment

30. DeVita P, Hortobágyi T. Obesity is not associated with increased knee joint torque and power during level walking. J Biomech. 2003;36(9):1355-1362; doi: 10.1016/ S0021-9290(03)00119-2.

31. Freedman Silvernail J, Milner CE, Thompson D, Zhang S, Zhao X. The influence of body mass index and velocity on knee biomechanics during walking. Gait Posture. 2013;37(4):575-579; doi: 10.1016/j.gaitpost.2012.09.016.

32. Lai PPK, Leung AKL, Li ANM, Zhang M. Three-dimensional gait analysis of obese adults. Clin Biomech. 2008; 23(Suppl 1):S2-S6; doi: 10.1016/j.clinbiomech.2008. 02.004 .

33. Cupp T, Oeffinger D, Tylkowski C, Augsburger S. Agerelated kinetic changes in normal pediatrics. J Pediatr Orthop. 1999;19(4):475-478; doi: 10.1097/00004694199907000-00010.

34. Dusing SC, Thorpe DE. A normative sample of temporal and spatial gait parameters in children using the GAITRite electronic walkway. Gait Posture. 2007; 25(1):135-139; doi: 10.1016/j.gaitpost.2006.06.003.

35. Ganley KJ, Powers CM. Gait kinematics and kinetics of 7-year-old children: a comparison to adults using age-specific anthropometric data. Gait Posture. 2005; 21(2):141-145; doi: 10.1016/j.gaitpost.2004.01.007.

36. Holm I, Tveter AT, Feredriksen PM, Vøllestad N. A normative sample of gait and hopping on one leg parameters in children 7-12 years of age. Gait Posture. 2009;29(2):317-321; doi: 10.1016/j.gaitpost.2008.09.016.

37. Ounpuu S, Gage JR, Davis RB. Three-dimensional lower extremity joint kinetics in normal pediatric gait. J Pediatr Orthop. 1991;11(3):341-349; doi: 10.1097/01241398199105000-00012.

38. Sutherland DH, Olshen RA, Biden EN, Wyatt MP. The development of mature walking. Oxford: Mac Keith Press; 1988.

39. McKay MJ, Baldwin JN, Ferreira P, Simic M, Vanicek N, Wojciechowski E, et al. Spatiotemporal and plantar pressure patterns of 1000 healthy individuals aged 3-101 years. Gait Posture. 2017;58:78-87; doi: 10.1016/j. gaitpost.2017.07.004.

40. Lythgo N, Wilson C, Galea M. Basic gait and symmetry measures for primary school-aged children and young adults whilst walking barefoot and with shoes. Gait Posture. 2009;30(4):502-506; doi: 10.1016/j.gaitpost. 2009.07.119. 